\title{
The Vimos-VLT Deep Survey: Results from the First-Epoch Observations
}

\author{
S. Paltani ${ }^{1}$, O. Ilbert ${ }^{1}$, O. Le Fèvre ${ }^{1}$, C. Marinoni ${ }^{1}$, D. Bottini ${ }^{2}$, B.
} Garilli $^{2}$, V. Le Brun ${ }^{1}$, D. Maccagni ${ }^{2}$, J.-P. Picat ${ }^{7}$, R. Scaramella ${ }^{4}$, M. Scodeggio $^{2}$, L. Tresse ${ }^{1}$, G. Vettolani ${ }^{4}$, A. Zanichelli ${ }^{4}$, C. Adami ${ }^{1}$, M. Arnaboldi $^{5}$, S. Arnouts ${ }^{1}$, S. Bardelli ${ }^{3}$, M. Bolzonella $^{6}$, A. Cappi ${ }^{3}$, S. Charlot $^{8,10}$, T. Contini ${ }^{7}$, S. Foucaud ${ }^{2}$, P. Franzetti $^{2}$, I. Gavignaud ${ }^{7,12}$, L. Guzzo ${ }^{9}$, A. Iovino ${ }^{9}$, H.J. McCracken ${ }^{10,11}$ B. Marano ${ }^{6}$, A. Mazure ${ }^{1}$, B. Meneux ${ }^{1}$, R. Merighi ${ }^{3}$, R. Pellò ${ }^{7}$, A. Pollo ${ }^{9}$, L. Pozzetti $^{3}$, M. Radovich $^{5}$, G. Zamorani ${ }^{3}$, E. Zucca ${ }^{3}$, M. Bondi ${ }^{4}$,A. Bongiorno ${ }^{6}$, G. Busarello $^{5}$, P. Ciliegi ${ }^{3}$, L. Gregorini ${ }^{4}$, G. Mathez ${ }^{7}$, Y. Mellier ${ }^{10,11}$, P. Merluzzi $^{5}$, V. Ripepi ${ }^{5}$, and D. Rizzo ${ }^{7}$

${ }^{1}$ Laboratoire d'Astropysique de Marseile, BP 8, 13376 Marseille Cedex 12, France

${ }^{2}$ IASF-INAF, via Bassini 15, 20133, Milano, Italy

${ }^{3}$ INAF-Osservatorio Astronomico di Bologna - Via Ranzani 1, I-40127, Bologna, Italy

${ }^{4}$ IRA-INAF, Via Gobetti 101, 40129, Bologna, Italy

${ }^{5}$ INAF-Osservatorio Astronomico di Capodimonte, via Moiariello 16, I-80131, Napoli, Italy

${ }^{6}$ Università di Bologna, Dipartimento di Astronomia, via Ranzani 1, 40127, Bologna, Italy

${ }^{7}$ LAOMP, 14 av. E. Belin, F31400 Toulouse, France

${ }^{8}$ Max Planck Institut fur Astrophysik, 85741 Garching, Germany

${ }^{9}$ INAF-Osservatorio Astronomico di Brera, via Brera 28, Milan, Italy

${ }^{10}$ Institut d'Astrophysique de Paris, 98 bis bvd Arago, 75014 Paris, France

${ }^{11}$ Observatoire de Paris, LERMA, 61 av. de l'Observatoire, 75014 Paris, France

${ }^{12}$ ESO, Karl-Schwarzschild-Strasse 2, 85748 Garching bei München, Germany

\begin{abstract}
The Vimos-VLT Deep Survey is a spectroscopic survey aiming at collecting more than 50000 spectra down to a limiting magnitude $I_{\mathrm{AB}}=24$, and 100000 down to $I_{\mathrm{AB}}=22.5$, on a total of about $16 \mathrm{deg}^{2}$ without any color or morphology preselection. We present the $N(z)$ distribution up to $z \sim 5$, obtained from a purely magnitude-limited sample down to $I_{\mathrm{AB}}=24$, which is an important input to weak-lensing studies. We discuss the evolution of the galaxy luminosity function up to $z \sim 2$, which exhibits a very strong increase in the typical galaxy luminosity $\Delta M^{*} \simeq-2.5$ in the U band compared to the local value. Surveys like the VVDS also allow to study the galaxy bias as a function of redshift without assumption about its linearity, an assumption that we find to be violated in some cases. A low bias is found, and the linear bias is shown to increase with redshift.
\end{abstract}

\section{The Vimos-VLT Deep Survey}

The Vimos-VLT Deep Survey (VVDS) is a spectroscopic survey dedicated to the measurement of more than 100000 galaxy redshifts in the range $0<z \leqslant 5$. The VVDS "wide" survey covers $16 \mathrm{deg}^{2}$ with a limiting magnitude $I_{\mathrm{AB}}=22.5$, and the VVDS "deep" survey covers about $1.3 \mathrm{deg}^{2}$ in the VVDS-02h field and $0.5 \mathrm{deg}^{2}$ in the Chandra Deep Field South (VVDS-CDFS). We concentrate here on the "deep" survey, for which about 11500 spectra have already been obtained on the VVDS-CDFS field (Le Fèvre et al. 2004a), and on the VVDS-02hr equatorial field (Le Fèvre et al. 2004b). 
The observations have been performed with the Vimos multi-object spectrograph, operating on the ESO-VLT3 Melipal telescope. Vimos allows the simultaneous observation of 600-800 objects in the spectral range 5500-9500 $\AA$ at a spectral resolution of $R \sim 230$. This resolution, together with other instrumental effects, allows a determination of the redshift with an accuracy $\Delta z \simeq 0.0009$. The spectroscopic survey is completed by a photometric survey in the B, V, R, and I filters (McCracken et al. 2003). Part of the VVDS-02hr has also been the target of $\mathrm{U}$, and/or J and K photometry.

The VVDS has been designed to provide very robust constraints on galaxy formation and evolution, and on the large scale structures over $90 \%$ of the life of the universe. The strength of the VVDS compared to other current deep surveys is the absence of color or morphological preselection, making the spectroscopic sample purely magnitude-limited. We present here some of the first results of the VVDS.

\section{Redshift distribution}

The redshift distribution of field galaxies, $N(z)$, is a very important observable. In the context of weak lensing, the reconstruction of the projected mass relies upon assumptions about this distribution. The VVDS can therefore provide very important constraints through the determination of $N(z)$ in a magnitude-limited sample of galaxies.

Redshift determination based on spectroscopy is however not always straightforward. While spectra presenting strong features like emission lines allow a fast and accurate redshift measurement, the redshift of other objects, either faint, presenting weak features, or marred by instrumental problems like fringing, can be very difficult to determine. As a result, less reliable redshifts have been attributed to about $13 \%$ of the galaxies, and no redshift at all to about $7 \%$ of them.

Previous redshift surveys have been plagued by the so-called "redshift desert", a range of redshifts between $z=1.5$ and $z=2.8$ where redshift determination is made particularly difficult by the combination of the absence of emission line, the weakness of the absorption lines, and the strong $\mathrm{OH}$ sky emission features. By doing an important work on the analysis of the spectral features at rest wavelength below $3700 \AA$ (Paltani et al. 2004), we have been able to cross partially this redshift desert.

Fig. 1 shows the redshift distribution for the 10000 galaxy redshifts obtained so far down to a limiting magnitude $I_{\mathrm{AB}}=24$. It can be seen that unreliable redshifts significantly affect only the domain $z>1.5$, where their fraction reaches $60 \%$ (compared to $12 \%$ for $z<1.5$ ). In spite of this, we find an important redshift tail at $z>2.8$. We stress that, while there are numerous unreliable redshifts in this redshift domain, the number of secure redshifts is still quite high compared to surveys using Lyman-break preselection techniques (e.g., Steidel et al. 1996). A detailed comparison of the high-redshift galaxy population density found in different surveys is presented in Paltani et al. (2004).

Many galaxies are found between $z=1.5$ and $z=2.2$, showing that we have succeeded in bridging more than half of the redshift desert. On the other hand, there is a clear dearth of redshifts in the range $2.2 \leqslant z \leqslant 2.8$. Vimos is unfortunately unable to probe this redshift domain in the configuration used in the VVDS. Part of the gap can also result from the $k$-correction which, for many galaxies, reaches a maximum in this spectral region (Paltani et al. 2004).

\section{Evolution of the luminosity function}

The luminosity function (LF) of galaxies is a very important diagnostic of the physical processes at play in the formation and evolution of galaxies. While the local LF is already 

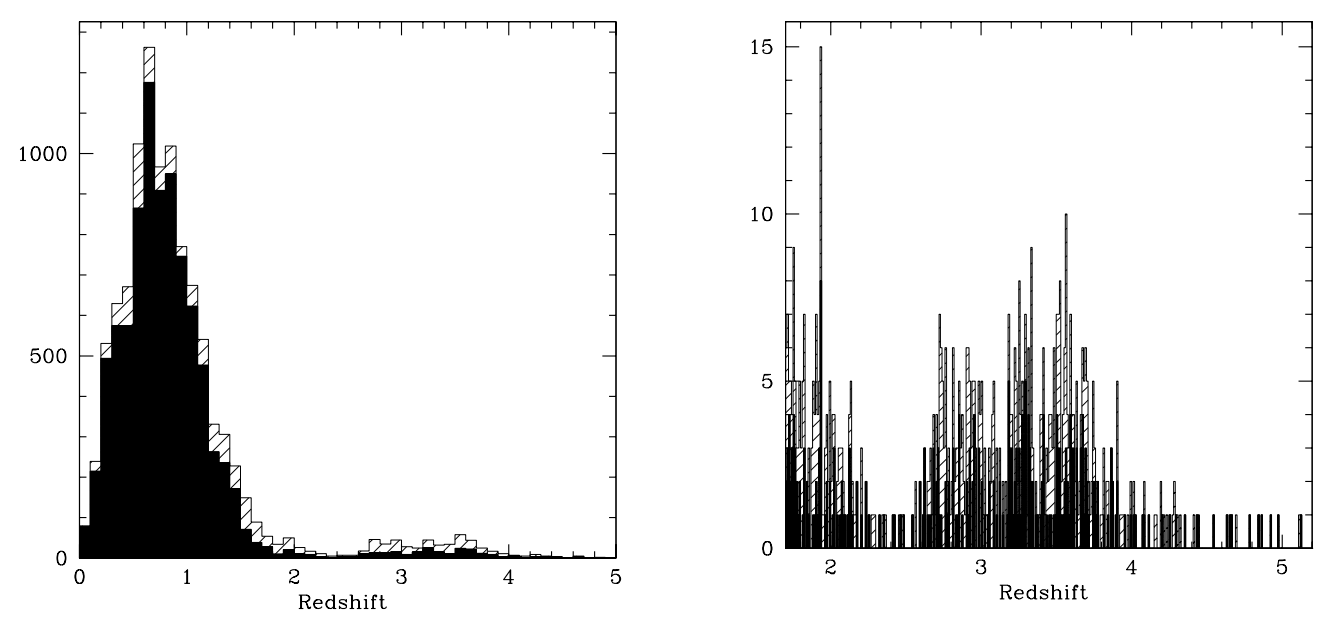

Figure 1. Left: Redshift distribution $N(z)$ between $z=0$ and $z=5$ in the VVDS-02h and VVDS-CDFS fields. The black area is a histogram of all secure redshifts; the dashed area includes the less reliable redshifts. Right: Zoom in the region $1.7 \leqslant z \leqslant 5$. Only the VVDS-02h data are included.

very well constrained by large spectroscopic surveys like the Two-Degree Field redshift Survey (2dFGRS; Norberg et al. 2002) and the Sloan Digital Sky Survey (SDSS; Blanton et al. 2001), the properties of the LF at $z>1$ are still uncertain. Thanks to the large number of galaxies detected at $z>1$, the VVDS allows to extend the study of the global LF towards redshifts up to $z=2$. Details on the determination of the global LF can be found in Ilbert et al. (2004b). The LF as a function of type is addressed in Zucca et al. (2004).

We determine the LF using four different estimators: $1 / V_{\max }$ (Schmidt 1968), $C^{+}$ (Zucca et al. 1997), STY (Sandage et al. 1979), and SWML (Efstathiou et al. 1988). Ilbert et al. (2004a) discuss the different biases affecting them, and define, as a function of redshift, the luminosity above which these biases can be neglected. Fig. 2 shows the $\mathrm{LF}$ in the $\mathrm{U}$ band from $z=0.05$ up to $z=2$. This band has been chosen because it is less affected by uncertainties in the $k$-correction. In the range $1<z<2$, the rest-frame $\mathrm{U}$ band is indeed redshifted very close to the I band, which renders the $k$-corrections very small, irrespectively of the galaxy types. Therefore the U-band LF is bound to be the most accurate LF at $z>1$. In Fig. 2, a weight has been applied to correct the LF for the effect of the target sampling rate, i.e. the fact that very extended sources have lower probability to be targeted spectroscopically, and for the effect of the spectroscopic success rate, i.e. the fact that only insecure redshifts could be attributed to a significant fraction of the sources (see $\S 2$ ). The latter correction has been performed by determining the redshift distribution of these objects using photometric redshifts in the area for which $\mathrm{U}, \mathrm{B}, \mathrm{V}, \mathrm{R}, \mathrm{I}, \mathrm{J}$, and $\mathrm{K}$ photometry is available (Ilbert et al. 2004b).

We find that the local LF is in very good agreement with that obtained by the SDSS (Blanton et al. 2001). The VVDS local LF is slightly steeper than the SDSS one (especially in the B band). As the VVDS reaches luminosities 2.5 magnitudes fainter than the SDSS, the VVDS slope is much better constrained, although the sampled volume is about 1000 times smaller in the VVDS. The characteritic luminosity $M^{*}$ is however not constrained, and had to be set to the SDSS value. 


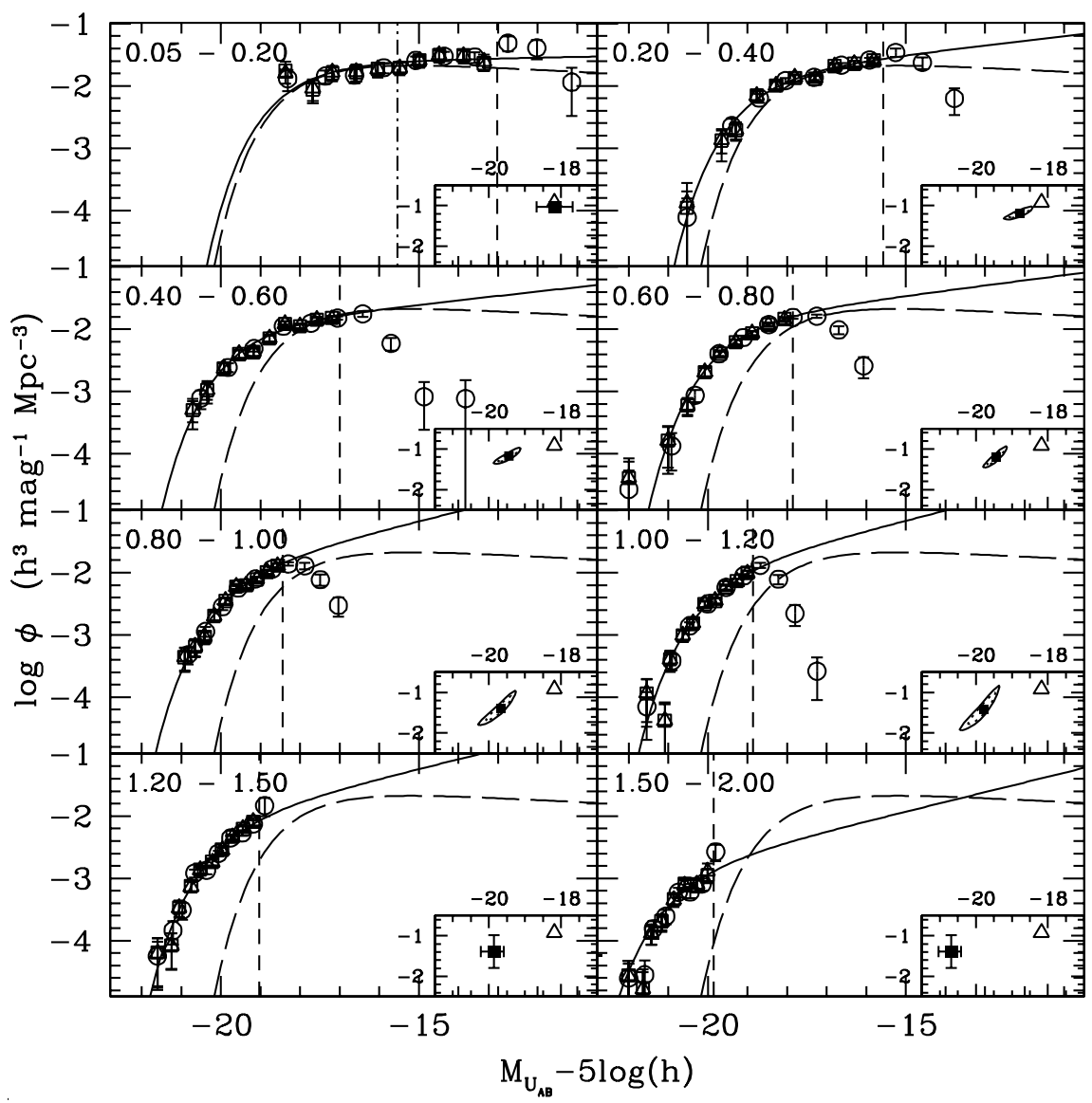

Figure 2. U-band luminosity functions in eight redshift bins. The circle, square, and triangle symbols have been computed using respectively the $1 / V_{\max }, C^{+}$, and SWML estimators. The solid line is the STY estimator. The heavy long-dashed line is the local luminosity function from the SDSS. The vertical long-dashed line is the limiting absolute magnitude above which the estimators become biased. The dot-dashed line in the first panel is the limiting absolute magnitude of the SDSS. In each panel, an inset shows the best fit parameters $\alpha$ and $M^{*}$. The triangles show the results from the SDSS.

Another important result is the evolution of $M^{*}$, the characteristic galaxy luminosity, which strongly increases with redshift. This is particularly important in the U-band LF, where $M^{*}$ decreases by $\sim 2.5$ magnitudes. By comparison, $M^{*}$ decreases by $\sim 1.6$ magnitudes when calculated in the I band. This is an indication that this evolution principally affects star-forming galaxies (Zucca et al. 2004).

\section{Galaxy bias}

The determination of the relationship between the galaxy density contrast with respect to the mean galaxy distribution, $\delta_{g}$, and the mass density contrast with respect to the cosmic density field, $\delta_{m}$, is one of the most important applications of lensing in cosmology. The linear bias, $b_{\mathrm{L}}$, is a constant defined under the assumption of a linear relation between these two quantities:

$$
\delta_{g}=b_{\mathrm{L}} \delta_{m}
$$



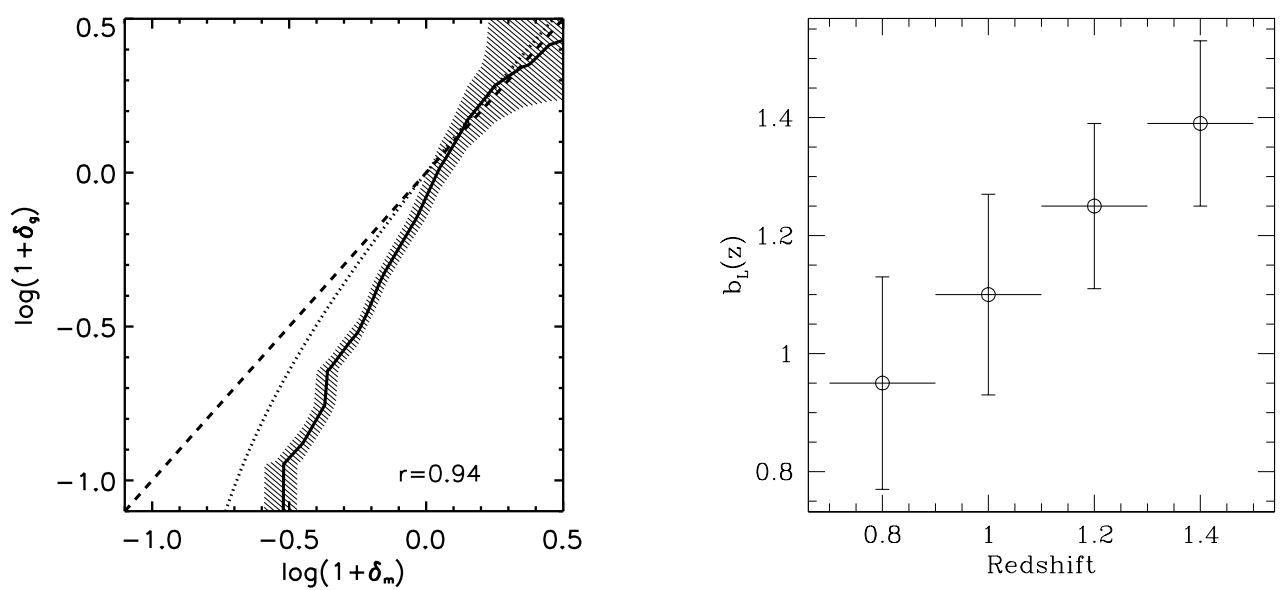

Figure 3. Left: Biasing function for galaxies with $M_{B}<-20.75+5 \log h$ in the redshift bin $0.9 \leqslant z \leqslant 1.1$ for density fluctuations smoothed on a scale $R=8 \mathrm{~h}^{-1} \mathrm{Mpc}$. The shaded area represents the $1 \sigma$ uncertainties. The dashed line is the unbiased relationship $\left(b_{\mathrm{L}}=1\right)$; the dotted line shows a perfectly linear bias with $b_{\mathrm{L}}=1.1$. Right: Redshift evolution of the linear bias for galaxies with $M_{B}<-20.75+5 \log h$ for density fluctuations smoothed on a scale $R=8 \mathrm{~h}^{-1} \mathrm{Mpc}$. The horizontal bars indicate the redshift bins.

However, in a more realistic scenario, the bias can be expected to be a complicated function of the redshift $z$, of the amplitude of mass fluctuations $\delta_{m}$, and of the smoothing scale $R$ on which the fluctuations are determined:

$$
\delta_{g}=b\left(z, \delta_{m}, R\right) \delta_{m}
$$

While the scale dependence can be easily investigated with weak lensing, the dependence with the redshift is tricky to determine, and requires a very good knowledge of the redshift distribution of galaxies. In addition, the dependence on $\delta_{m}$, i.e. the non-linearity of the bias, can possibly be determined only through the use of higher-order statistics of weak lensing. As a consequence, while weak lensing is probably the only method being able to trace the mass distribution, alternative methods of bias determination may prove to be necessary.

The VVDS provides an opportunity to address the problem of the bias. As $\delta_{m}$ cannot be directly measured from spectroscopic surveys like the VVDS, we take an orthogonal approach (Marinoni et al. 2004): We simply derive the PDF of mass fluctuations $f\left(\delta_{m}, z, R\right)$ from theory (e.g., Coles \& Jones 1991), and compare it to the observed PDF of VVDS galaxy fluctuations $g\left(\delta_{g}, z, R\right)$ directly in redshift space. Once the galaxy and mass PDFs are known, we can derive the biasing relationship $\delta_{g}\left(\delta_{m}, z, R\right)=b\left(z, \delta_{m}, R\right) \delta_{m}$ by solving the differential equation for a given redshift $z$ and a given scale $R$ :

$$
\frac{\mathrm{d} \delta_{g}\left(\delta_{m}, z, R\right)}{\mathrm{d} \delta_{m}}=\frac{f\left(\delta_{m}, z, R\right)}{g\left(\delta_{m}, z, R\right)} .
$$

A unique solution is obtained by imposing as an initial condition the physical requirement that galaxies cannot form in absence of mass. The advantage over other methods is that we can explore the functional form of the relationship $\delta_{g}=b\left(z, \delta_{m}, R\right) \delta_{m}$ over a wide range in mass density contrasts, redshift intervals, and smoothing scales $R$ without specifying any a priory parametric functional form for the biasing relationship $b\left(z, \delta_{m}, R\right)$. 
Fig. 3 left shows an example of the biasing function, defined as the relationship between $\log \left(1+\delta_{g}\right)$ and $\log \left(1+\delta_{m}\right)$, determined in the redshift bin $0.9 \leqslant z \leqslant 1.1$, for density flutuations smoothed on a scale $R=8 \mathrm{~h}^{-1} \mathrm{Mpc}$. We find evidence of non-linearities, as shown by the low correlation coefficient $r=0.94$. The bias is stronger for low mass density fluctuations $\delta_{m}$, and there is a hint of a decrease in the bias at high $\delta_{m}$. From the more general biasing relation $b\left(z, \delta_{m}, R\right)$, the linear bias can be retrieved by averaging $b\left(\delta_{m}\right)$ (Dekel \& Lahav 1999):

$$
b_{\mathrm{L}}(R, z)=\frac{<b\left(\delta_{m}, R, z\right) \delta_{m}^{2}>}{<\delta_{m}^{2}>} .
$$

Fig. 3 right shows the evolution of the linear bias for galaxies with $M_{B}<-20.75+5 \log h$ over the cosmic domain probed by the VVDS on a scale $R=8 \mathrm{~h}^{-1} \mathrm{Mpc}$. There is a clear evolution in the sense that the bias increases by about $50 \%$ between $z \sim 0.8$ and $z \sim 1.5$.

\section{Conclusions}

An important new result of the VVDS is the discovery of a strong high-redshift tail in a $I_{\mathrm{AB}}=24$ magnitude-limited sample. While its study is still on-going, even counting only secure redshifts implies a large galaxy population density at $z>3$. The global luminosity function of galaxies shows a very strong increase of the characteristic luminosity in the rest-frame ultraviolet with redshift, which is probably associated with more intense starformation in the past. The VVDS data allow us to study the galaxy bias without any assumption about its dependence on the mass density or redshift; this study is therefore a very interesting complement to bias studies based on weak lensing. We find the bias to be quite low, with evidence of an increase with redshift; there is also evidence of non-linear bias.

\section{Acknowledgements}

This research has been developed within the framework of the VVDS consortium. This work has been partially supported by the Italian Ministry (MIUR) grants COFIN2000 (MM02037133) and COFIN2003 (num.2003020150).

\section{References}

Blanton M.R. et al. 2001, ApJ 121, 2358.

Coles P. \& Jones, B. 1991, MNRAS 248, 1.

Dekel A. \& Lahav O. 1999, ApJ 520, 24.

Efstathiou G., Ellis R.S. \& Peterson B.A. 1988, MNRAS 232, 431.

Ilbert O. et al. 2004a, MNRAS 351, 541.

Ilbert O. et al. 2004b, A\&BA, Submitted.

Le Fèvre O. et al. 2004a, A\&BA, Submitted.

Le Fèvre O. et al. 2004b, A\&AA, Submitted.

Marinoni C. et al. 2004, A\&SA, Submitted.

McCracken H.J. et al. 2003, $A \& A$ 410, 17.

Norberg P. et al. 2002, MNRAS 336, 907.

Paltani S. et al. 2004, A\&A, Submitted.

Schmidt M. 1968, ApJ 151, 393.

Steidel C.C., Giavalisco M., Pettini M., Dickinson M. \& Adelberger K.L. 1996, ApJ 462, L17.

Zucca E. et al. 1997, A\&SA 326, 477.

Zucca E. et al. 2004, A\&SA, Submitted. 